\title{
Hybrid technopolitics for a collective construction
}

\section{SIGRADI2018 TECHNOPOLITICAS \\ xxii congresso da sociedade iberoamericana de gráfica digital 22th conference of the iberoamerican society of digital graphics $07|08| 09$ |novembro|2018 iau usp | são carlos $\mid \mathrm{sp}$ br}

\author{
Patricia Muñoz \\ Universidad de Buenos Aires | Argentina | patricia@plm.com.ar
}

\section{Analía Sequeira}

Universidad de Buenos Aires | Argentina | analiasequeira.di@gmail.com

María Varela

Universidad de Buenos Aires | Argentina | mariavarelamazza@hotmail.com

\begin{abstract}
This paper refers to the creation of a collective construction as the opening class of three correlative courses. Before the event, the installation was designed and planned by the teaching staff, combining digital and analogical instruments, which allowed us to carry out a project that involved over five hundred participants. On the site, students built the objects without digital tools, as they were unavailable. These hybrid resources made possible the production of an object that was meaningful for both, students and teachers, as an introductory action of the concepts that would be developed in the courses throughout the year.
\end{abstract}

Keywords: Hybrid; Installation; Collaborative; Education; Restrictions.

\section{INTRODUCCIÓN}

Frente a condicionantes y restricciones en la disponibilidad de recursos en la enseñanza superior pública, diseñamos estrategias para generar actividades que combinen los medios con los que contamos, tanto analógicos como digitales, incrementando progresivamente su com- plejidad. Acordamos con Carolina León (1995) cuando sostiene: "La tecnología no es buena ni mala per se. Está implicada en un contexto tanto de producción como de aplicación" (p.48). Por esto, entendemos que las tecnopolíticas pueden referirse también a la mixtura de instrumentos tradicionales e innovadores.

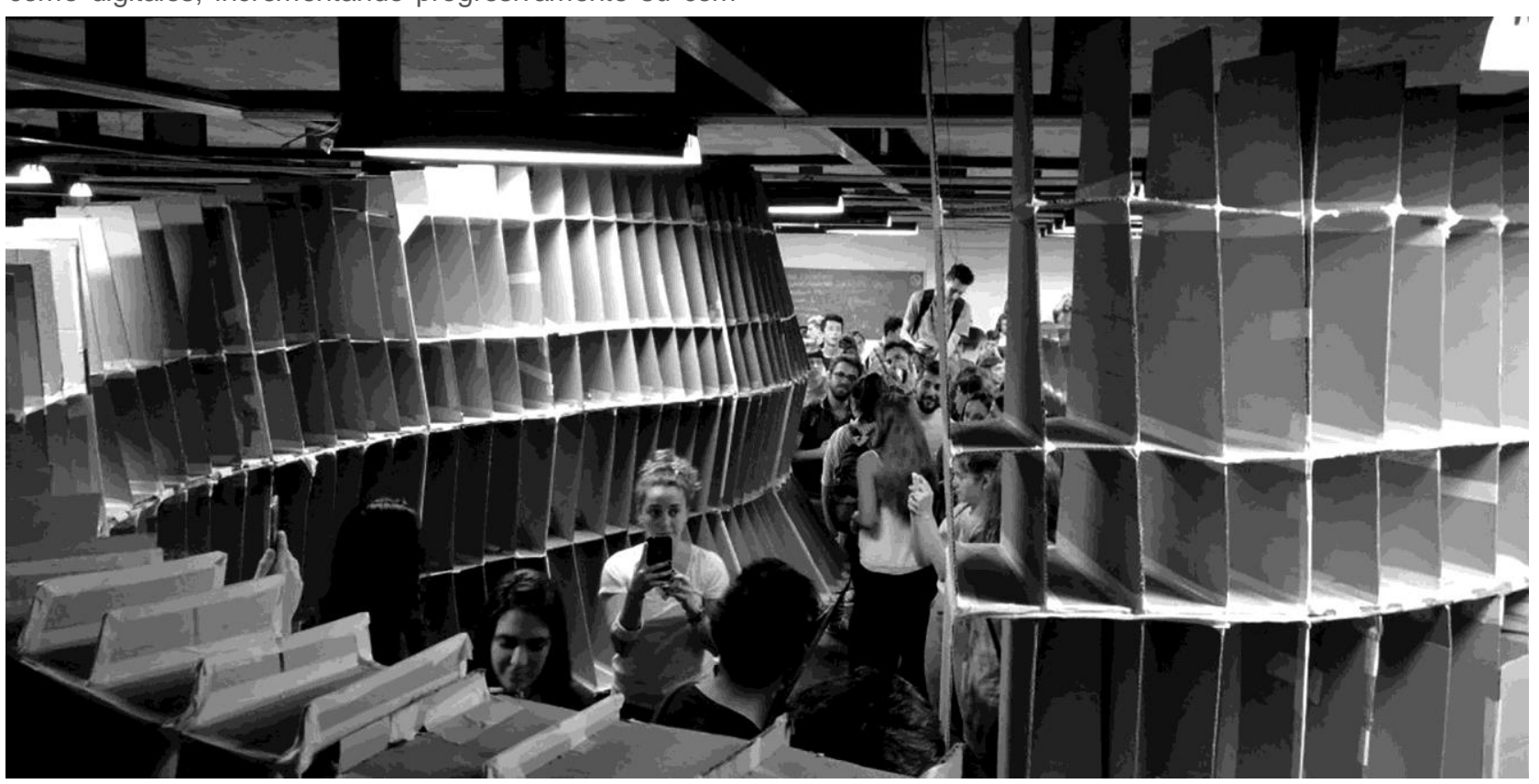

Figura 1: Imagen general de la instalación con los estudiantes. Fuente: Autores. 
En esta oportunidad, describiremos el trabajo inaugural, producido por treinta docentes y más de quinientos alumnos de tres niveles de las asignaturas de Morfología, Cátedra Muñoz, de la Carrera de Diseño Industrial de la Facultad de Arquitectura, Diseño y Urbanismo de la Universidad de Buenos Aires, Argentina (Figura 1). El desafío consistió en construir, de modo colaborativo y en menos de cuatro horas, un artefacto que promoviera un recorrido espacial, con muy bajo costo económico y sin contar con herramientas informáticas durante la actividad. Esto fue posible integrando los medios digitales en el diseño y planificación del objeto, como así también en la logística y en la producción de la información para que los estudiantes lo construyeran, coordinados por los docentes.

En la cátedra valoramos la realización de acciones conjuntas, trascendiendo las intervenciones individuales. Propiciamos esta actividad en consonancia con estas ideas, para que puedan manifestarse en una obra concreta y visible. Desde hace varios años, el primer día de clase de nuestros cursos, se realiza una práctica inicial que consiste en una construcción colectiva. (Figura 2) Cada curso como grupo, de más de cien alumnos y seis docentes, produce objetos referidos a los contenidos, que constituyen material didáctico orientado a futuras prácticas (Muñoz, 2006). El proceso de fabricación de las formas facilita la comprensión y la visualización de su sistema generativo, como así también que se verifiquen sus elementos constitutivos y su estructura. Es una experiencia muy motivadora ya que los estudiantes se vuelven protagonistas de un trabajo colectivo, que logra resultados impactantes a través de una actividad de baja complejidad y laboriosidad, con un costo casi despreciable.

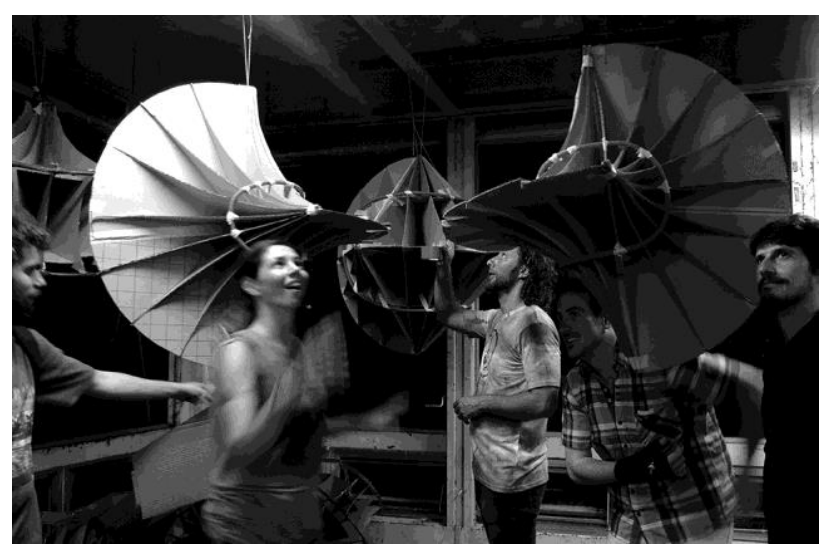

Figura 2: Trabajos iniciales Curso 2015. Fuente: Autores.

Hasta la realización de este artefacto, nunca habíamos intentado integrar los tres cursos - ya que se trataría de más de trescientos alumnos- ni habíamos abordado un objeto de esta escala, que pudiera ser recorrido por los estudiantes. Estas acciones colectivas aprovechan las ventajas de la enseñanza superior masiva, ya que brindan a docentes y alumnos la posibilidad de contar con un espacio y tiempo compartido para producciones que serían inviables en grupos reducidos. Asimismo, hace frente a las limitaciones de recursos, propios de la Universidad pública, encontrando alternativas de producción que se realizan no a pesar de los límites sino con los límites. Acordamos con Prigogine y Stengers (cita en Manzini, 1990) cuando plantean: "Un condicionamiento no limita simplemente lo posible, sino que también es oportunidad; no se impone simplemente, desde el exte- rior, a una realidad ante todo existente, sino que participa en la construcción de una estructura integrada y, según el caso, determina un espectro de consecuencias nuevas e inteligibles"(p.107).

\section{METODOLOGÍA}

\section{ANÁLISIS DE REQUERIMIENTOS Y DE PRODUCCIO- NES SIMILARES}

Se planteó al equipo docente la inquietud de realizar en la práctica inaugural de los cursos, un objeto construido de modo colaborativo por docentes y estudiantes, con las siguientes características:

- Debía ser autoportante, y construido por módulos que pudieran trasladarse desde el lugar de construcción al sitio de armado.

- Debía tener una escala que permitiera recorrerlo, planteando instancias espaciales diferentes. La altura máxima era de $2.70 \mathrm{~m}$, considerando que es la del techo de los talleres. Si bien no estaba definido el lugar de armado (patio central, pasillo, taller), debido a los permisos necesarios, se consideró la instancia de menor altura.

- Para limitar el gasto de los estudiantes, convenía realizarse con placas de cartón corrugado de $1 \mathrm{x}$ $0,70 \mathrm{~m}$ y cinta de embalaje, y no debía requerir más que una placa por integrante del curso.

- Se contaba con medios digitales para el desarrollo del proyecto y del material explicativo previo a la realización, pero para la construcción los recursos serían solamente analógicos.

- Como se realizaba en un solo encuentro, el tiempo máximo a emplear en la construcción era de tres horas ya que la clase completa es de cuatro.

Se analizaron experiencias semejantes, como los producidos en el Tongji International Construction Festival, 2015, Shanghai, y otras que se consideraron de especial relevancia, como el muro plegable de cartón de Holabalsa, y el Lat Pavillion del FAB13.

\section{DISEÑO Y REALIZACIÓN MIXTURADA: ANALÓGICO- DIGITAL}

Con posterioridad, se realizaron varios encuentros presenciales. El equipo de diseño exploró diferentes opciones para la práctica, algunas de ellas pueden verse en la Figura 3. Se plantearon alternativas que partían de encastres entre piezas, tanto en dos como en tres dimensiones. El trabajo inicial fue sobre maquetas a escala, en cartulina, con instancias de mayor rigurosidad con dibujos en CAD. Finalmente, se optó por una propuesta que toma una de las restricciones-las medidas del cartón- como idea de diseño. El artefacto contaría con tres niveles horizontales, compuestos por placas perpendiculares al piso, uno apaisado y dos verticales, de modo de llegar a una altura de 2,70m como máximo. La disposición de las placas seguiría el sistema generativo de la forma diseñada, acompañando las rotaciones. 


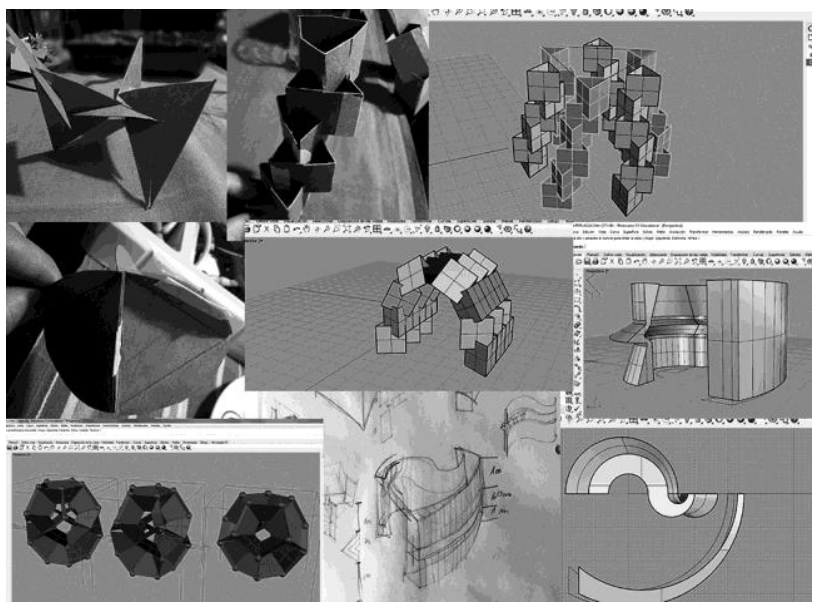

Figura 3: Desarrollo de alternativas. Fuente: Autores.

A partir de estos criterios, y de prefiguraciones que se sucedían, tanto en croquis como en CAD; parte del grupo docente generó un proyecto conformado por dos muros curvos enfrentados, que planteaban una experiencia de entrada, recinto y pasillo al recorrerlo. El exterior del objeto alternaba concavidades y convexidades de modo continuo, mientras que en el interior esto se manifestaba de modo discontinuo (Figura 4).

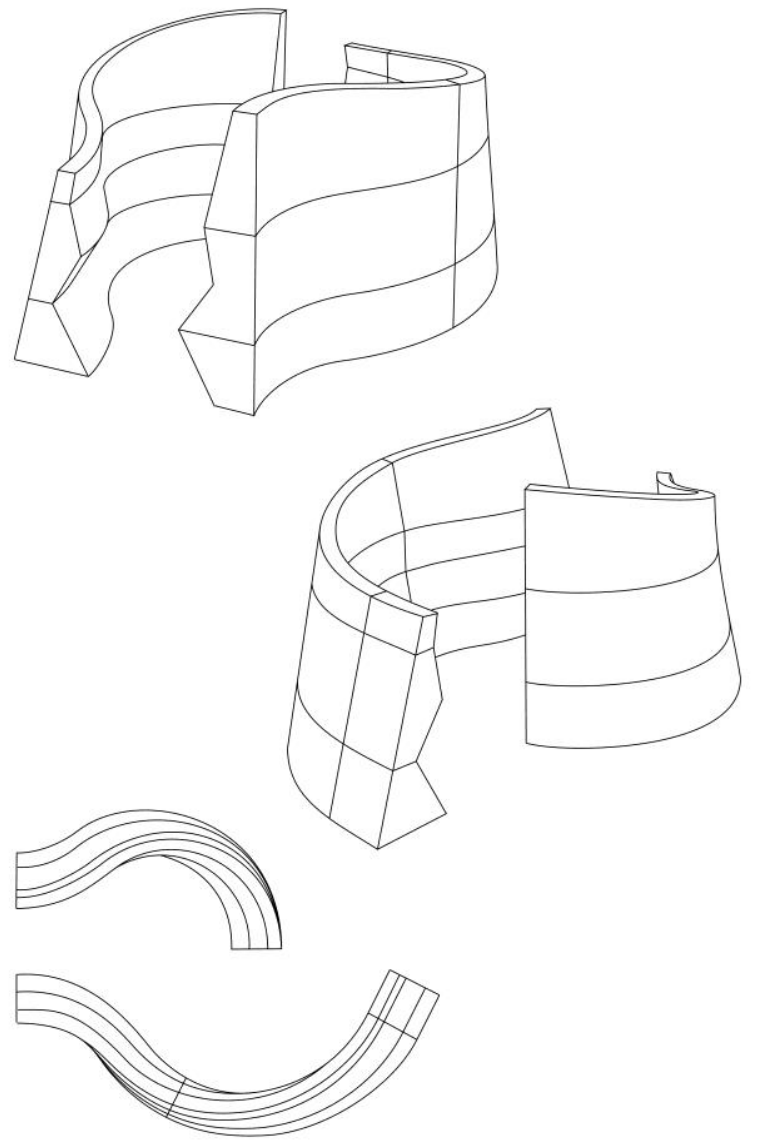

Figura 4: Propuesta final. Fuente: Autores.

Ambos muros estaban concretados por una constitución sistemática y cada estudiante produciría una pieza a partir del corte de una placa de cartón corrugado. Esta limitación condicionó y favoreció la organización de la

materialización, ya que se estableció como unidad de medida, que no sólo determinó las alturas de los tres niveles horizontales, sino que también definió la división en módulos verticales, ya que debían ajustarse a sus medidas máximas. Así, el objeto quedó conformado por veintiún módulos verticales, cada uno compuesto por tres bloques.

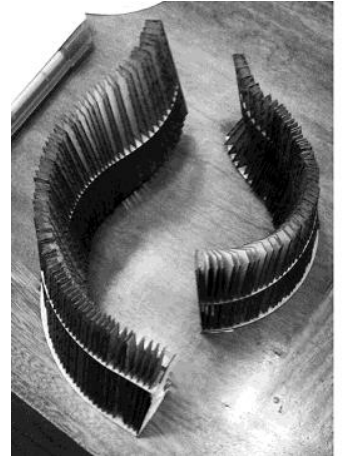

Figura 5: Maqueta de estúdio general y de los bloques. Fuente: Autores.

Se modeló el objeto en 3D, y se cortó en láser una maqueta de estudio 1:10. Se verificó la validez de la propuesta, se ajustaron detalles y se definieron las piezas que conformarían cada uno de los bloques (Figura 5). Se realizó también una grilla para establecer la cantidad de material necesario por módulo. Se asignó a cada curso un sector, encargándole al coordinador del nivel la entrega de la documentación a cada docente a cargo de grupo para su construcción. Ésta consistía en las imágenes de las piezas que constituían el bloque y sus elementos estructurantes, en escala 1:10. Cada pieza se presentó sobre una retícula con las proporciones del cartón (Figura 6 ). Por otra parte, el layout se amplió por medio de cuadrículas a partir de una impresión a escala, para llevarlo a la medida real como guía para la ubicación de los módulos el día del armado. Si bien esto podría haberse ploteado, en función de seguir manteniendo el costo bajo y de replicar las acciones que llevarían adelante los estudiantes, se decidió hacerlo a mano.
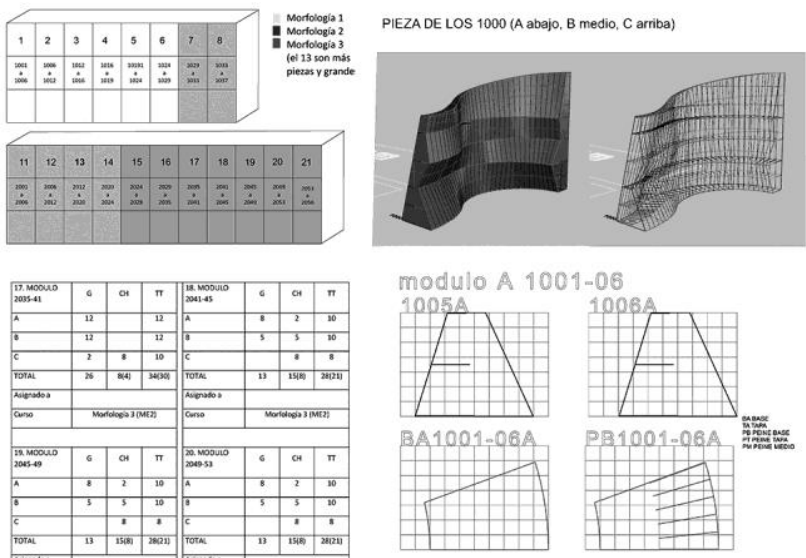

Figura 6: Grillas organizativas y ejemplos del material brindado a los estudiantes. Fuente: Autores. 
En el aula se asignaron los materiales por grupos de estudiantes (aproximadamente de 25 integrantes cada uno), que debían organizarse para comprar el material, distribuir tareas de ampliación, dibujo, corte y armado, para lograr construir el sector asignado. El trabajo se realizó a mano, por lo tanto se presentaron esperables imprecisiones. Sin embargo, previo al montaje, se producía una verificación y ajuste de la variación de las piezas verticales en el grupo, para que esta ambigüedad se redujera de modo de no impactar significativamente en el producto final. Estas acciones no sólo eran relevantes en función de mejorar la apariencia del resultado, sino también para que el estudiante comprendiera con mayor profundidad, que su participación cobraba sentido en la acción colectiva, que reconocía imprecisiones y las regulaba para que no fueran significativas. Algunas pocas piezas se volvieron a cortar para evitar discontinuidades indeseadas por error, a partir de la decisión del grupo de estudiantes. Si bien el objeto final presenta inexactitudes dimensionales, consideramos que no son relevantes a los fines de la actividad.

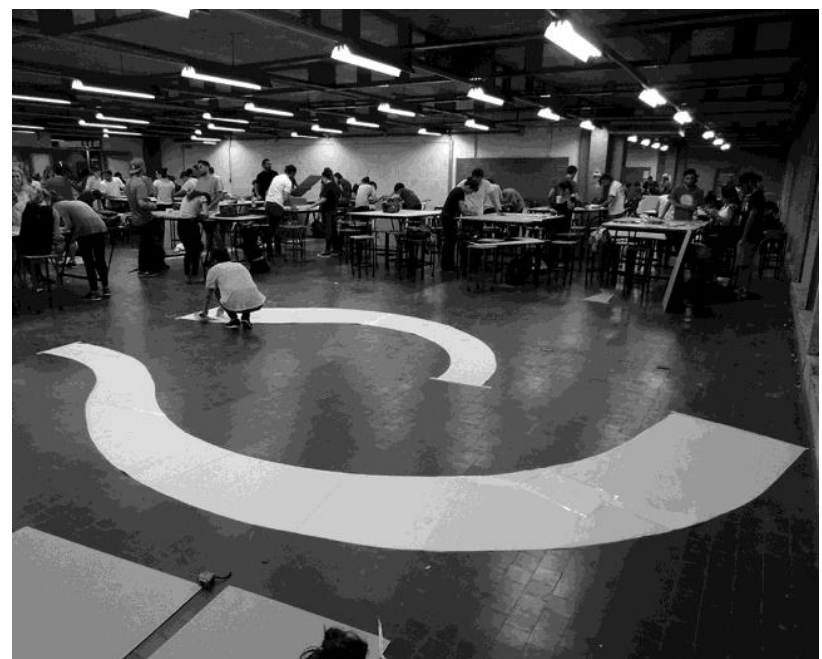

Figura 7: Layout e início del armado. Fuente: Autores.

Fue de particular importancia que los módulos fueran autoportantes, ya que esta actividad se desarrolló en 6 aulas, 3 en el primer piso y 3 en el entrepiso superior y debían moverse por escaleras al aula de armado definitivo. Empleando el layout 1:1 se buscó la mejor ubicación
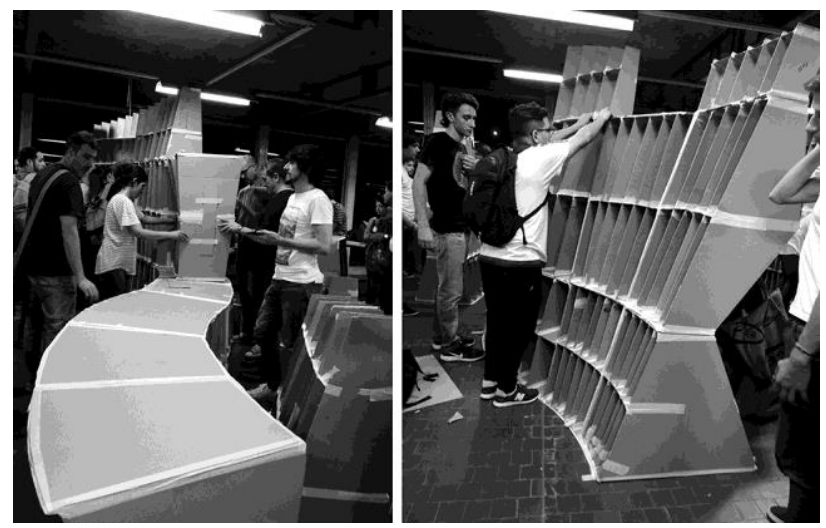

Figura 8: Etapas del armado. Fuente: Autores. para facilitar el montaje final. Fue de gran ayuda ya que estaba indicada la posición de cada módulo (Figura 7).

Fue fundamental la coordinación de los coordinadores de cada curso para convocar a los constructores de cada bloque ordenadamente. Por razones estructurales, se armó primero el nivel del piso y cuando estuvo completo se fue subiendo a los siguientes (Figura 8).

\section{RESULTADOS}

Se podría plantear que el resultado de la práctica fue el objeto final. Entendemos que es el resultado más visible pero no el único. Por un lado, la forma obtenida, su sistema generativo y su lógica constructiva están vinculados a los contenidos específicos de la materia, anticipando la comprensión de temas que se desarrollarán en los cursos. Por otro, no podemos dejar de considerar que facilitó el establecimiento de relaciones interpersonales entre estudiantes que no se conocían, que seguirán trabajando a lo largo del año en nuestros cursos (Figura 9). Consideramos también, de gran valor, que los alumnos pasaran por la experiencia de integrar un grupo de trabajo tan numeroso, de diferente procedencia y formación, con el objetivo de realizar una acción común que iba haciéndose visible paulatinamente en su concreción.

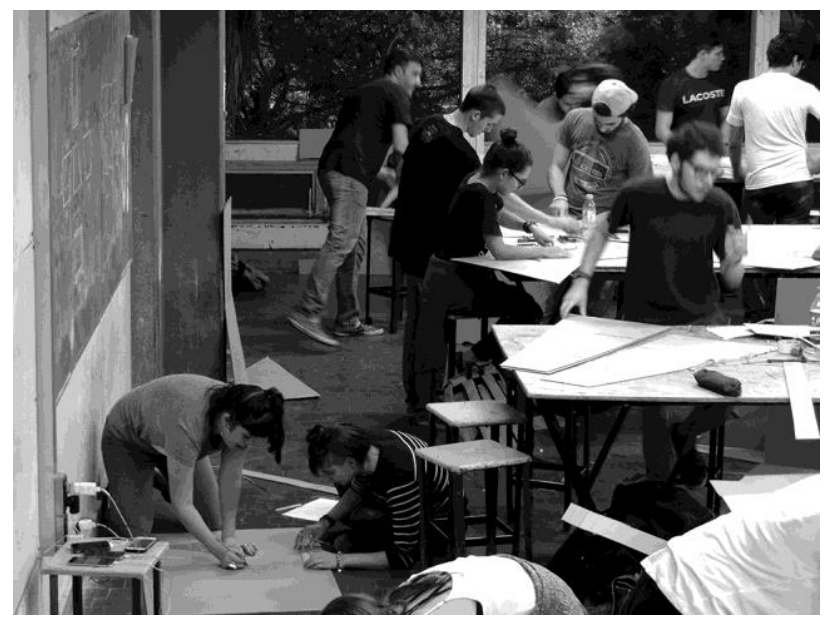

Figura 9: Clima de trabajo del primer día del curso. Fuente: Autores.

La asignatura está dividida en tres cursos, Morfología 1 a 3 , que si bien coinciden en el mismo día y horario, se desarrollan en diferentes espacios físicos (aunque próximos). Esto no facilita la percepción de la unidad de sentido que tiene el dictado de la materia en su concepción, definida por el enfoque de los contenidos abordados, por la noción de forma en el contexto del diseño que elaboramos y por las modalidades didácticas que promovemos. La construcción colectiva de este objeto vinculó, a través de las acciones, a los estudiantes y docentes de los tres cursos, produciendo una primera manifestación de esta unidad de sentido.

\section{DISCUSIÓN}

$\mathrm{Ni}$ los medios digitales ni los analógicos por si mismos hubieran hecho viable esta experiencia en nuestro contexto. Es en la interacción donde aparecen nuevas posibilidades productivas y generativas. 
Si hubiera sido todo digital, la actividad de los participantes hubiese consistido solamente en el armado del objeto. De ese modo se hubiera eliminando la sensibilización de los estudiantes para encontrar las características significativas de la forma de la pieza a ampliar. Este conocimiento les permitía manejar una pequeña tolerancia con respecto a la medida nominal, que garantizara la continuidad en la visualización de las transformaciones del producto final. Este umbral es equiparable a la inexactitud del croquis, que presenta ambigüedades en la precisión de sus proporciones y sus trazos pero que permiten visualizar la acción proyectual.

Podríamos haber sumado más recursos digitales en el despiece de las formas. La falta de tiempo para incorporar estas herramientas en los plazos necesarios hizo que no se hiciera de modo automático y que aumentara la cantidad de tiempo dedicado a esta acción.

Esto mismo es lo que plantean nuestros estudiantes cuando proponemos y propiciamos la integración de nuevos recursos digitales, como Grasshopper, que es más eficiente -en precisión y en el tiempo requerido-, al momento de resolver algunas cuestiones que los alumnos deben abordar en sus cursos. A lo largo de los años, fuimos desarrollando material de apoyo, tutoriales, breves prácticas limitadas a aspectos operativos, con la intención de revertir esto. Si bien hemos logrado un avance significativo (de $11 \%$ de estudiantes que lo usaron en 2014 a un $48 \%$ en 2017) queda mucho por hacer. Sin embargo, los comentarios de quienes lo usaron fueron positivos, tales como: "Fueron muy útiles y ahorraron mucho tiempo", "Una herramienta útil en la que puedo seguir explorando", "Sirvió para calcular las medidas exactas y generar una forma adecuada”. (Cátedra Muñoz, 2015)

Las explicaciones más frecuentes sobre la falta de uso son las mismas que nosotros empleamos para no abordar otros recursos digitales que, si bien requerían mayor inversión de tiempo en la etapa inicial, hubieran representado una reducción en la dedicación una vez superada. En las encuestas los estudiantes también se refirieron a este obstáculo, con comentarios tales como: "Perdía más tiempo aprendiendo a usarlo que usando otro programa que ya conocía", "Por falta de tiempo no quise meterme con algo nuevo", "No tuve tiempo para experimentar", etc.
Si hubiera sido todo a mano, el requerimiento de tiempo dedicado y la laboriosidad lo hubiese tornado inviable. De haber decidido avanzar igual en este camino, en lugar de realizarse la documentación por dos docentes hubiera sido necesario involucrar a los treinta, con mayores posibilidades de acumular errores en la división de tareas. Nuestras primeras experiencias de construcciones conjuntas como trabajo inicial, se realizaron con recursos digitales muy limitados. Por esto también, eran producciones reducidas en cuanto a cantidad de alumnos y componentes involucrados por proyecto.

A partir de esto surge otro análisis. Frente a la programación de las asignaturas, con los contenidos que se desarrollarán, es muy relevante definir cuánto tiempo se va a dedicar a una acción colectiva de este tipo, que si bien aborda aspectos vinculados a los contenidos de los tres niveles, requiere mucha dedicación en su formulación. Se busca el delicado equilibrio en la organización curricular, ya que aquello nuevo que se incluye exige que otro contenido se excluya de la práctica, por la limitación de la planificación del tiempo de clases. En este caso en particular, entendemos que los beneficios son significativos, y superan los del desarrollo de contenidos específicos, como para dedicarle la primera clase de los tres cursos a esta actividad, con el trabajo previo que implica. Superamos el desafío de cambiar la escala del objeto y de los participantes en esta construcción conjunta, valiéndonos de todos los recursos disponibles en la mixtura analógicadigital que consideramos apropiada.

Asumimos un riesgo. Sabíamos que podían surgir imponderables. Sin embargo, era reducido ya que contábamos con el conocimiento de haber realizado experiencias similares, aunque menores, en años previos. Pudimos abordar la experiencia y valió la pena. Dos indicadores fueron significativos. Por el lado de los estudiantes, ellos se quedaron un tiempo más, al terminar la actividad, para sacarse fotos con el objeto y filmar recorridos que compartieron en sus redes sociales (Figura 10). Por parte del grupo docente, esto también sucedió. Además, ese mismo día, ya estábamos compartiendo y considerando alternativas e instancias superadoras para el año próximo. Si bien puede parecer anticipado, es una clara muestra del entusiasmo que provocan estos desafíos.

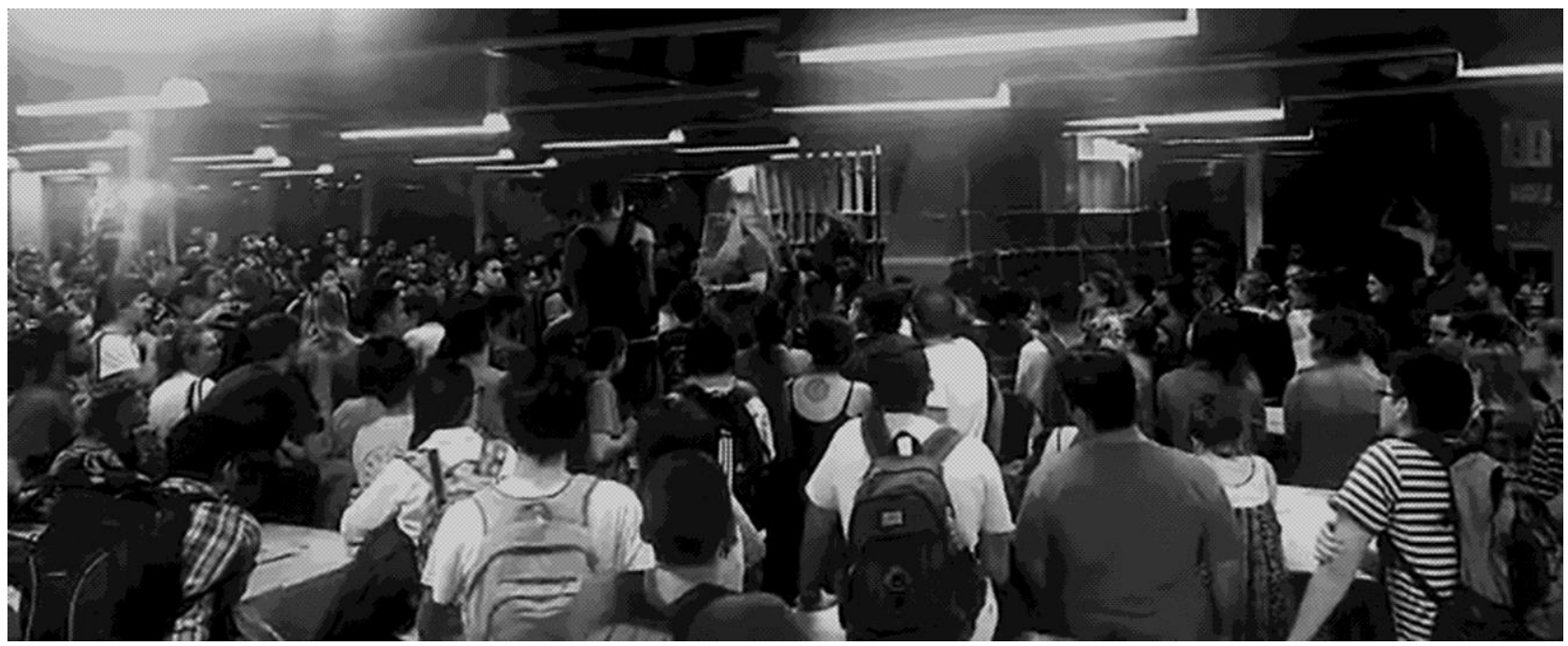

Figura 10: Cierre de la clase, con la producción y los participantes. Fuente: Autores. 


\section{REFERENCIAS}

Holabalsa (n.d.) Recuperado de https://www.facebook.com/ holabalsa/videos/1587557511300824/ https://www.facebook.com/holabalsa/

Lion, C. G. (1995) Mitos y realidades en la tecnología educativa. En Litwin, E. (comp.)Tecnología educativa (pp.41-64). Buenos Aires: Paidos

Manzini, E. (1990) Artefactos, Madrid: Celeste Ediciones y Experimenta Ediciones de Diseño.

Muñoz, P. (2006) Capítulo 6. Morfología y medios digitales, En Carmena, S. (coord.) Experiencia digital. Usos, prácticas y estrategias en talleres de arquitectura y diseño en entornos virtuales (pp. 101-111). Mar del Plata; EUDEM - Diana Rodríguez Barros.

Cátedra Muñoz (2015) Encuestas de estudiantes 2014. Recuperado de http://www.plm.com.ar/academico

Pabellón LAT (n.d.) Recuperado de http://www.fablabconnect.com/lat-pavilion-pabellon-latcompleted-fab13/

Tongji International Construction Festival (n.d.) Recuperado de http://tjdi.tongji.edu.cn/NewsDetail.do?ID=3800\&lang=en y https://mp.weixin.qq.com/s? biz=MjM5Mzl2MjU2MQ\%3D\% $3 D \&$ mid $=200336779 \& i d x=1 \& s n=b c 46 e b 9 a 243 d 22 a 7 f c 51 a 7 d$ $2 \mathrm{a} 011 \mathrm{a} 65 \mathrm{~b} \&$ scene $=1 \&$ from $=$ singlemessage $\&$ isappinstalled $=$ 0\#rd 